Full length article

\title{
Ontogenetic changes in foot strike pattern and calcaneal loading during walking in young children
}

\author{
Angel Zeininger ${ }^{\mathrm{a}, *}$, Daniel Schmitt ${ }^{\mathrm{a}}$, Jody L. Jensen ${ }^{\mathrm{b}}$, Liza J. Shapiro ${ }^{\mathrm{c}}$ \\ a Department of Evolutionary Anthropology, Duke University, Box 90383, Durham, NC, 27708-0383, USA \\ b Department of Kinesiology and Health Education, The University of Texas at Austin, 2109 San Jacinto Blvd., Stop D3700, Austin, TX, 78712, USA

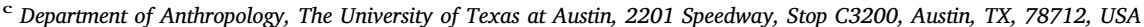

\section{A R T I C L E I N F O}

\section{Keywords:}

Bipedalism

Ground reaction force

Center of pressure

Heel strike

\begin{abstract}
A B S T R A C T
The assumption that the morphology of the human calcaneus reflects high and cyclical impact forces at heel strike during adult human walking has never been experimentally tested. Since a walking step with a heel strike is an emergent behavior in children, an ontogenetic study provides a natural experiment to begin testing the relationship between the mechanics of heel strike and calcaneal anatomy. This study examined the ground reaction forces (GRFs) of stepping in children to determine the location of the center of pressure (COP) relative to the calcaneus and the orientation and magnitude of ground reaction forces during foot contact. Three-dimensional kinematic and kinetic data were analyzed for 18 children ranging in age from 11.5 to 43.1 months. Early steppers used a flat foot contact (FFC) and experienced relatively high vertical and resultant GRFs with COP often anterior to the calcaneus. More experienced walkers used an initial heel contact (IHC) in which GRFs were significantly lower but the center of pressure remained under the heel a greater proportion of time. Thus, during FFC the foot experienced higher loading, but the heel itself was relatively wider and the load was distributed more evenly. In IHC walkers load was concentrated on the anterior calcaneus and a narrower heel, suggesting a need for increased calcaneal robusticity during development to mitigate injury. These results provide new insight into foot loading outside of typical mature contact patterns, inform structure-function relationships during development, and illuminate potential causes of heel injury in young walkers.
\end{abstract}

\section{Introduction}

It is assumed that the robust calcaneus of adult humans is adapted to withstand high, cyclical impact forces during walking and that these forces have shaped its morphology both during our evolution and during ontogeny [1]. For example, the robust calcaneal tuber with a prominent lateral plantar process is thought to provide a wide base of support over which ground reaction forces (GRFs) can be dissipated during adult heel strike [1]. Heel contact patterns develop gradually throughout bipedal development [2-9] but the lateral plantar process appears early in development, as the lateral plantar cornu [1], suggesting either that heel contact forces are the same throughout ontogeny or that the lateral plantar process develops in the absence of routine loading. Additionally, young children, some at the earliest stages of walking, experience calcaneal fractures and damage to the apophyseal plate [10-12], suggesting that high loading may occur in the absence of a well-defined heel strike. Yet little is known about changes in calcaneal loading throughout development, leaving functional features and clinical aspects of development unexplored. Such data are needed to address questions regarding the functional anatomy of the human calcaneus.

In adult walking, the heel makes initial contact with the ground at the end of swing phase and the foot and leg experience an impact transient [13-16]. Simultaneous vertical (Fz) and horizontal (fore-aft; Fy) forces result in an upward and backward (posteriorly) oriented braking GRF resultant (GRFr). The center of pressure (COP), which serves analytically as the anchor point of the GRFr, lies beneath the heel during heel strike in adults [17-20]. As such, the adult heel pad and underlying calcaneus experience, and must mitigate, relatively high magnitude GRFs that project through the hind foot at heel strike.

Hallemans et al. [4,5,21] showed that at the earliest stages of locomotor development, young children exhibit flat foot contact (FFC), in which the heel, midfoot, and metatarsals simultaneously experience peak pressure at touchdown. At later stages of development, children use an initial heel contact (IHC), during which peak pressure is roughly under the heel at touchdown $[4,5,21]$. However, little is known about

\footnotetext{
* Corresponding author at: Department of Evolutionary Anthropology, Duke University, Box 90383, Durham, NC, 27708, USA.

E-mail addresses: azeininger@gmail.com, angel.zeininger@duke.edu (A. Zeininger), daniel.schmitt@duke.edu (D. Schmitt), jljensen@austin.utexas.edu (J.L. Jensen), liza.shapiro@austin.utexas.edu (L.J. Shapiro).
} 

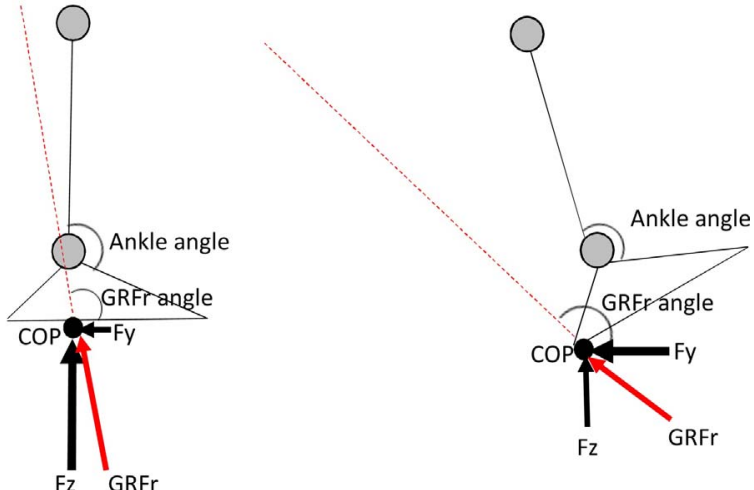

Fig. 1. Models of flat foot contact and initial heel contact. In the flat foot contact (FFC) model, the ankle angle is relatively large and the foot is placed beneath the body at touchdown. The center of pressure (COP) is beneath the ankle and the horizontal (braking) ground reaction force (Fy) is small compared to the vertical ground reaction force (Fz). In the initial heel contact (IHC) model, the ankle angle is relatively small (dorsiflexed) and the foot is placed anterior to the whole body center of mass at touchdown. The COP is beneath the heel and the braking force (Fy) is large compared to the vertical force (Fz). Red arrows represent the magnitude and angle of the resultant ground reaction force (GRFr) and dotted red lines are projections of the GRFr. (For interpretation of the references to colour in this figure legend, the reader is referred to the web version of this article.)

the peak forces and impact transients experienced during these early stages of walking and how they may affect calcaneal morphology. In this study, we investigate how initial heel contact configuration (FFC and IHC) influences the position of the COP and the magnitude and orientation of the GRFr at touchdown, at the time of vertical impact peak, and at the time of braking peak.

Infant stepping is characterized by high degrees of hip and knee flexion [3] resulting in a hip lift and foot drop with each step. We hypothesized that this vertical landing path, along with minimal ankle dorsiflexion, leads to an FFC that positions the COP close to the vertical projection of the whole body center of mass at touchdown. Thus, in FFC steps, we predicted that this vertical transfer of a large proportion of total body weight results in a relatively high vertical ground reaction force (Fz) but relatively low horizontal (braking) GRF (Fy) (i.e., the ratio of Fy/Fz is small) (Fig. 1). Further, as the sagittal orientation of the GRFr (GRFr angle) is a function of the magnitude of the Fy and Fz, we predicted that the GRFr would project only slightly posterior in FFC steps. Early walkers (with less than 6 months walking experience) might also be expected to have relatively higher mediolateral (Fx) GRFs because of wide steps [22] and abducted hips [23]. Therefore, we calculated Fx forces, but Fy and Fz forces were the focus of our study, as they are most relevant to distinguishing between FFC and IHC foot strike patterns.

In contrast, to achieve initial heel contact (IHC), we hypothesized that a long stride allows the leg to swing through a large arc and, coupled with an adducted hip [23] and a dorsiflexed ankle, positions the heel as the point of contact at touchdown. In this model, the heel contacts the ground relatively far in front of the whole body center of mass, compared to FFC steps, and the COP is under the heel at touchdown (Fig. 1). With this more forward position of the COP relative to the body, a relatively lower proportion of total body weight is immediately transferred to the stance foot. As such, we predicted that for IHC walkers, 1) mediolateral (Fx) and vertical GRF (Fz) would be relatively low compared to FFC steps and 2) horizontal (Fy, braking) GRF would be relatively high (i.e., the ratio of $\mathrm{Fy} / \mathrm{Fz}$ is large). If $\mathrm{Fy} / \mathrm{Fz}$ differs between foot strike patterns, then the angle of the GRFr must also differ, with a more posteriorly directed GRFr predicted for IHC compared to FFC steps. Since a wide base of the calcaneal tuber is thought to be an adaptation to heel strike in adults, we predicted that,
Table 1

Child walking data.

\begin{tabular}{|c|c|c|c|c|c|c|c|}
\hline Subject & age (months) & $\begin{array}{l}\text { months } \\
\text { walking }\end{array}$ & $\begin{array}{l}\text { body } \\
\text { mass } \\
(\mathrm{kg})\end{array}$ & $\begin{array}{l}\text { hip } \\
\text { height } \\
(\mathrm{cm})\end{array}$ & $\begin{array}{l}\text { heel } \\
\text { length: } \\
\text { foot } \\
\text { length }\end{array}$ & $\begin{array}{l}\text { heel } \\
\text { length: } \\
\text { heel } \\
\text { width }\end{array}$ & steps \\
\hline \multicolumn{8}{|l|}{ IHC } \\
\hline 8 & 16.8 & 5.6 & 7.3 & 32.8 & 0.34 & 0.75 & 3 \\
\hline 12 & 30.2 & 17.2 & 11.4 & 38.7 & 0.31 & 0.80 & 5 \\
\hline 9 & 33.8 & 20.3 & 13.9 & 38.5 & 0.31 & 0.94 & 5 \\
\hline 7 & 36.4 & 25.9 & 14.5 & 42.8 & 0.38 & 1.10 & 2 \\
\hline 1 & 38.7 & 24.2 & 14.5 & 45.5 & 0.31 & 0.86 & 2 \\
\hline 2 & 40.2 & 28.2 & 13.6 & 44.5 & 0.25 & 0.93 & 2 \\
\hline 6 & 43.1 & 35.1 & 14.1 & 42.6 & 0.34 & 1.00 & 5 \\
\hline Mean & 34.2 & 22.3 & 12.8 & 40.8 & 0.32 & 0.92 & \\
\hline & 8.7 & 9.4 & 2.6 & 4.41 & 0.04 & 0.12 & \\
\hline TOTAL & & & & & & & 24 \\
\hline \multicolumn{8}{|l|}{ FFC } \\
\hline 18 & 11.5 & 3.0 & 10.2 & 29.7 & 0.33 & 0.94 & 2 \\
\hline 3 & 11.7 & 0.5 & 10.0 & 31.5 & 0.31 & 0.84 & 2 \\
\hline 13 & 12.3 & 1.6 & 9.8 & 32.1 & 0.32 & 0.80 & 5 \\
\hline 15 & 12.7 & 1.7 & 10.9 & 30.6 & 0.30 & 0.76 & 2 \\
\hline 14 & 15.3 & 0.8 & 9.9 & 32.3 & 0.33 & 0.91 & 5 \\
\hline 17 & 15.8 & 3.0 & 11.4 & 31.9 & 0.31 & 0.72 & 2 \\
\hline 19 & 16.3 & 0.3 & 11.0 & 31.7 & 0.33 & 0.99 & 5 \\
\hline 10 & 16.9 & 4.9 & 11.1 & 31.5 & 0.25 & 0.65 & 5 \\
\hline 11 & 18.0 & 5.0 & 10.2 & 34.9 & 0.30 & 0.76 & 3 \\
\hline 4 & 21.5 & 7.5 & 11.9 & 39 & 0.25 & 0.86 & 4 \\
\hline 5 & 26.4 & 12.4 & 11.4 & 39.6 & 0.28 & 0.81 & 4 \\
\hline Mean & 16.2 & 3.7 & 10.7 & 33.2 & 0.30 & 0.82 & \\
\hline SD & 4.6 & 3.7 & 0.07 & 3.29 & 0.03 & 0.10 & \\
\hline TOTAL & & & & & & & 39 \\
\hline
\end{tabular}

IHC = initial heel contact. FFC $=$ flat foot contact. $\mathrm{SD}=$ standard deviation.

compared to children who use FFC, children who use IHC would have a relatively wide heel (i.e., the ratio of heel length to heel width would be small).

\section{Materials and methods}

Subject participation was approved by the Institutional Review Board, University of Texas at Austin, and informed consent was obtained from each participant's parent or legal guardian before participation. Cross-sectional data were collected on eighteen subjects (Table 1) at the Developmental Motor Control Laboratory in the Department of Kinesiology and Health Education at the University of Texas at Austin. Kinematic data were collected at $120 \mathrm{~Hz}$ via a 10 camera Vicon MX (Vicon, Centennial, CO) motion analysis system, synchronized with Bertec (Bertec, Columbus, $\mathrm{OH}$ ) force plates. Reflective markers $(6 \mathrm{~mm})$ were placed on one foot following a modified foot marker set of Stebbins and colleagues [24], specifically validated for measuring foot biomechanics in children. Lower limb length was measured as the distance from the hip to the ground when standing and body weight was measured with a standing spring balance.

Barefoot subjects walked, unassisted, at a self-selected speed over two adjacent force plates that recorded GRFs at $1200 \mathrm{~Hz}$. Trials in which only one foot was on the plate were reconstructed in Vicon Nexus 1.8.2. Raw kinematic and kinetic data were filtered using a fourth order zero-lag phase Butterworth low pass filter with a cut-off frequency of $12 \mathrm{~Hz}$ (kinematic) or $100 \mathrm{~Hz}$ (kinetic), as determined following methods of Winter [25].

Kinetic data were presented as the magnitude of the GRF vector in three dimensions $(\mathrm{Fz}=$ vertical, $\mathrm{Fy}=$ fore-aft, and $\mathrm{Fx}=$ mediolateral $)$ and the location of the COP (COPx, COPy). Vertical, horizontal, and mediolateral ground reaction forces $(\mathrm{N})$ were normalized to body weight (e.g., Fy/BW) and used to calculate the magnitude and direction of the GRFr. The GRFr angle was made a continuous variable $\left(0^{\circ}-180^{\circ}\right)$ by calculating the angle between the ground and the GRFr in the 


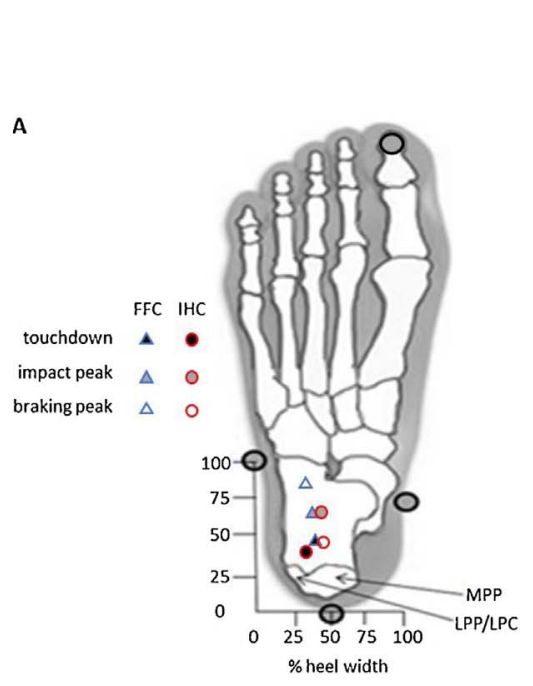

Initial heel contact
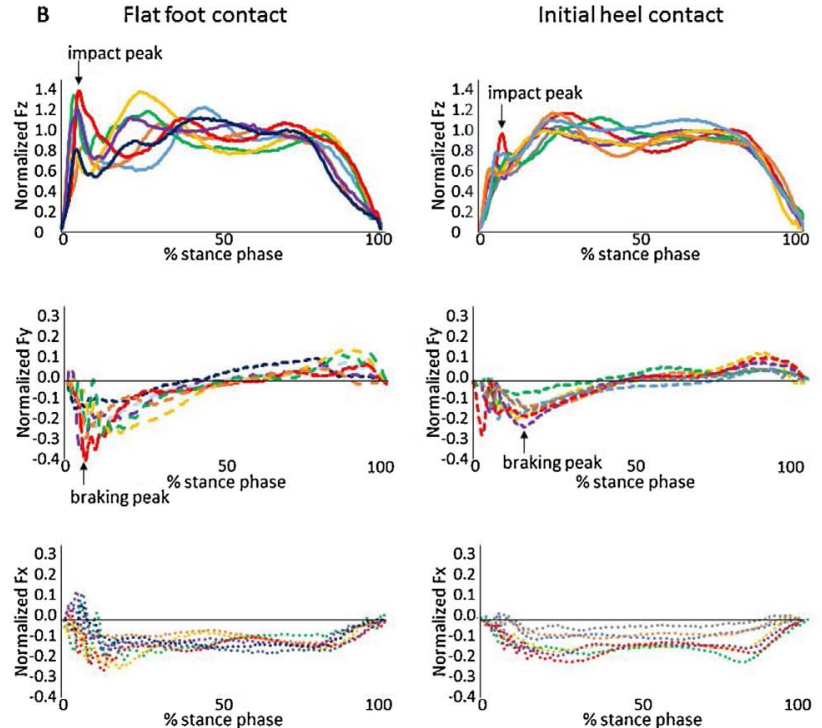

Adult heel strike
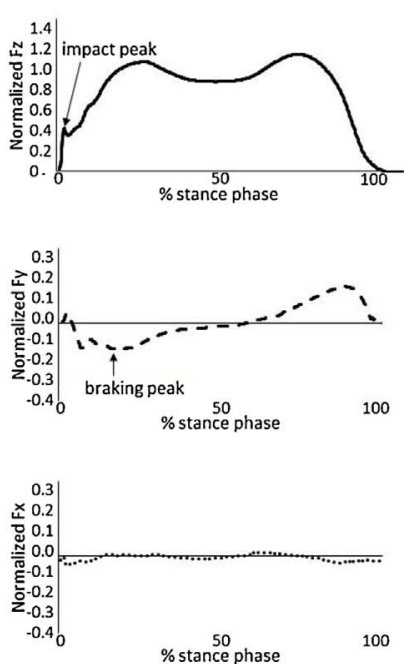

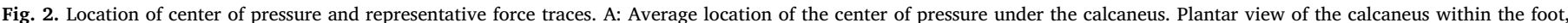

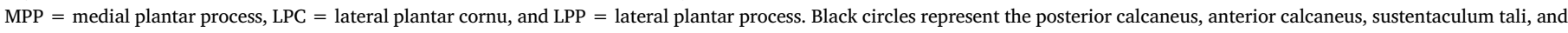

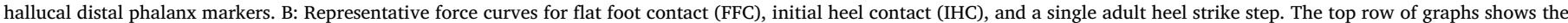

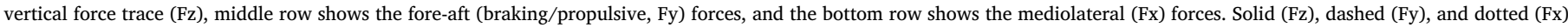

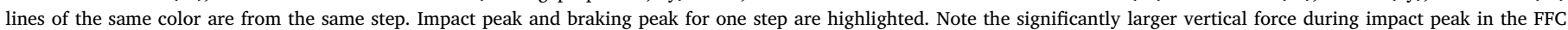
compared to the IHC steps. Also note that forces are highly variable, especially in FFC steps.

sagittal plane.

Three-dimensional marker coordinate data were used to calculate the distance between the COP and bony landmarks of the foot (Fig. 2), allowing projection of the GRFr throughout the hind foot (Fig. 3). Location of the COP under the heel was determined using markers on the posterior calcaneus, anterior calcaneus on the lateral side of the foot, and sustentaculum tali and was represented as \% heel length and \% heel width. The posterior calcaneus was set as $0 \%$ heel length and the anterior calcaneus was set as $100 \%$ heel length. The lateral calcaneus (aligned with the anterior calcaneus marker) was set as $0 \%$ heel width and the medial calcaneus (aligned with the sustentaculum tali marker) was set as $100 \%$ heel width. Given that the anterior calcaneus is elevated in initial heel contact, the antero-posterior position of the COP was measured from the posterior calcaneal marker. To test for differences in heel length between foot strike patterns, we calculated the ratio of heel length to foot length (posterior calcaneus to distal hallux). Relative heel width was calculated as the ratio of heel length to heel width for each subject.

Foot strike pattern was determined visually [15] by examining lateral video-recordings of each step. In FFC steps, the heel, midfoot, and metatarsal heads contacted the force plate simultaneously (i.e., only fully plantigrade was categorized as FFC). In IHC steps, the heel made initial contact with the force plate such that the midfoot was clearly elevated above the ground. An alternative method to classify foot strike pattern would be based on the relative position of the center of pressure. However, since an important aim of our study was to evaluate the influence of foot strike pattern on COP position, it would be circular to use COP position to define foot strike patterns. Although there are potential limitations associated with visual determination of foot strike patterns, we attained $100 \%$ inter-rater reliability in a $30 \%$ subsample of steps.

Values for COP position and GRFr magnitude and orientation were analyzed and compared between FFC and IHC at three points during early stance: touchdown, time of impact peak, and time of braking peak. Touchdown was defined as the moment the magnitude of the vertical GRF (Fz) reached or exceeded $10 \%$ of body weight. Impact peak occurred at the Fz maximum of the vertical GRF transient (i.e., the first Fz spike) and braking peak was the peak negative Fy value.

Significant differences between IHC and FFC steps were tested for using a $t$-test in Statistica 10 (www.statsoft.com). Although the number of steps differed between subjects, previous developmental studies favor an analysis of individual steps to reducing steps to a subject mean (in which case within group variability, rather than individual steps, is compared) $[26,27]$. To test for the effects of the subject, we iteratively removed one subject at a time in a leave-one-out sensitivity analysis; however, even though there was a high degree of variability between
Touchdown

FFC

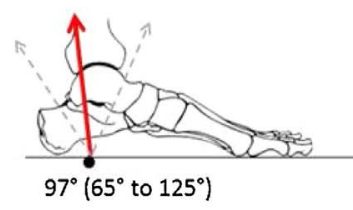

$\mathrm{IHC}$

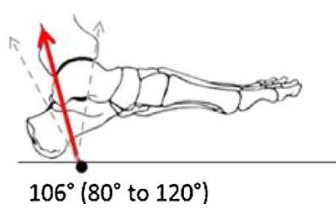

Impact Peak

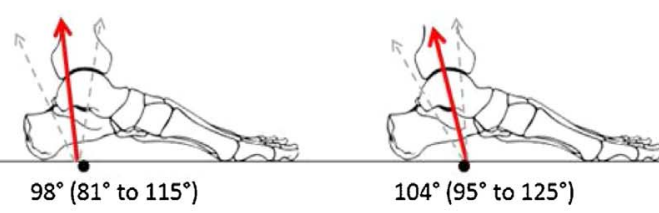

$98^{\circ}\left(81^{\circ}\right.$ to $\left.115^{\circ}\right)$

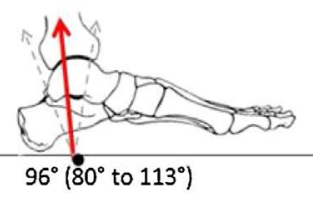

Braking Peak

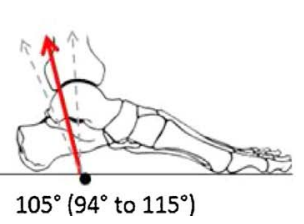

Fig. 3. Ground reaction force resultant (GRFr) angle during early stance phase. Mean GRFr angle is shown as the red arrow. Gray arrows show the minimum and maximum GRFr angle for each event in early stance phase in flat foot contact (top) and initial heel contact (bottom) steps. Location of the center of pressure (black circle, anchor point of the GRFr) is an approximation based on mean calcaneal length when the COP was under the calcaneus. Length of arrow does not represent GRFr magnitude. (For interpretation of the references to colour in this figure legend, the reader is referred to the web version of this article.) 


\begin{tabular}{|c|c|c|c|c|c|c|c|c|c|}
\hline \multirow[b]{2}{*}{ Ankle angle $\left({ }^{\circ}\right)$} & \multicolumn{3}{|l|}{ Touchdown } & \multicolumn{3}{|l|}{ Impact Peak } & \multicolumn{3}{|l|}{ Braking Peak } \\
\hline & $\begin{array}{l}\text { FFC } \\
108(7.8)\end{array}$ & $\begin{array}{l}\text { IHC } \\
107(7.5)\end{array}$ & ns & $\begin{array}{l}\text { FFC } \\
106(8.3)\end{array}$ & $\begin{array}{l}\text { IHC } \\
108(9.1)\end{array}$ & ns & $\begin{array}{l}\text { FFC } \\
103(9.19)\end{array}$ & $\begin{array}{l}\text { IHC } \\
106(8.8)\end{array}$ & ns \\
\hline \multicolumn{10}{|l|}{$\%$ of steps COP is: } \\
\hline under heel & 30 & 100 & & 8 & 46 & & 2 & 29 & \\
\hline $\begin{array}{l}\text { anterior to heel } \\
\text { Location of COP }\end{array}$ & 70 & 0 & & 92 & 54 & & 98 & 71 & \\
\hline$\%$ heel length & $48(32.3)$ & $40(23.6)$ & ns & $67(3.2)$ & $68(23.7)$ & ns & $84^{\mathrm{a}}$ & $48(15.9)$ & - \\
\hline$\%$ heel width & $40(14.7)$ & $32(15.0)$ & ns & 37 (3.9) & $41(8.5)$ & ns & $34^{\mathrm{a}}$ & $46(10.0)$ & - \\
\hline \multicolumn{10}{|c|}{ GRF magnitude (\%BW) } \\
\hline normalized $\mathrm{Fx}^{\mathrm{b}}$ & $0.02(0.01)$ & $0.01(0.01)$ & $\mathrm{p}=0.003$ & $0.09(0.06)$ & $0.06(0.05)$ & ns & $0.11(0.07)$ & $0.08(0.06)$ & ns \\
\hline normalized Fy & $-0.02(0.04)$ & $-0.04(0.03)$ & ns & $-0.12(0.11)$ & $-0.07(0.09)$ & ns & $-0.22(0.10)$ & $-0.17(0.07)$ & $\mathrm{ns}$ \\
\hline normalized Fz & $0.12(0.02)$ & $0.12(0.01)$ & ns & $0.90(0.30)$ & $0.69(0.30)$ & $\mathrm{p}=0.005$ & $0.89(0.32)$ & $0.67(0.25)$ & $p=0.007$ \\
\hline normalized Fy/Fz & $0.28(0.28)$ & $0.32(0.20)$ & ns & $0.16(0.11)$ & $0.13(0.11)$ & ns & $0.26(0.12)$ & $0.28(0.13)$ & ns \\
\hline normalized GRFr & $0.13(0.02)$ & $0.12(0.02)$ & ns & $0.92(0.30)$ & $0.70(0.30)$ & $\mathrm{p}=0.006$ & $0.91(0.33)$ & $0.69(0.25)$ & $p=0.006$ \\
\hline \multicolumn{9}{|l|}{ GRFr angle $\left({ }^{\circ}\right)$} & ns \\
\hline all steps & $97(16.4)$ & $106(11.3)$ & $\mathrm{p}=0.002$ & $98(7.2)$ & $96(7.5)$ & ns & $104(5.7)$ & $105(6.3)$ & $\mathrm{ns}$ \\
\hline
\end{tabular}

Means are presented with standard deviations in parentheses. Bold p values are statistically significant, ns = not significant. BW $=$ body weight.

${ }^{\text {a }}$ Sample size is too small to calculate a standard deviation or test for significant differences.

${ }^{\mathrm{b}}$ For normalized Fx, all values were made positive so that absolute magnitude of all mediolateral forces could be compared.

steps, results of the sensitivity analysis did not suggest that any one subject was significantly influencing the results.

\section{Results}

Sixty-three steps $(\mathrm{IHC}=24, \mathrm{FFC}=39$ ) were analyzed (Table 1$)$. Compared to children who used an FFC, IHC steppers were, on average older ( $\sim 34$ months), heavier ( $\sim 12 \mathrm{~kg}$ ), had longer legs ( $\sim 40 \mathrm{~cm}$ hip height), and had more walking experience ( $\sim 22$ months) (Table 1$)$. The ratio of heel length to foot length did not differ significantly between IHC and FFC subjects ( $\mathrm{p}>0.05$; Table 1 ). However, compared to FFC subjects, IHC subjects had a significantly higher heel length to heel width ratio $(\mathrm{p}=0.005$; Table 1$)$ indicating a more narrow heel.

At touchdown, impact peak, and braking peak, there were no significant differences in ankle angle between IHC and FFC ( $\mathrm{p}>0.05)$ (Table 2). Nevertheless, IHC and FFC steps differed significantly with respect to relative position of the COP and magnitude of loading. Specifically, the COP was located under the heel at touchdown in $100 \%$ of IHC steps but only $30 \%$ of FFC steps (Table 2). In the remaining $70 \%$ of FFC steps the COP was anterior to the heel. At impact peak, the COP was still under the heel in $46 \%$ of IHC steps but only $8 \%$ of FFC steps. The COP remained under the heel at braking peak in $29 \%$ of IHC steps but only $2 \%$ of FFC steps. When the COP was under the heel, there were no significant differences in COP location between FFC and IHC at touchdown or impact peak ( $p>0.05$ ) (Table 2). Small sample size for the COP under the heel at braking peak in FFC steps $(n=1)$ precluded statistical testing of COP location for this gait event.

Normalized vertical force (Fz) and GRFr magnitudes were significantly higher in FFC than IHC at impact peak $(\mathrm{p}<0.01)$ and braking peak ( $\mathrm{p}<0.01$ ) (Fig. 2; Table 2). In association with the relatively high vertical force, FFC subjects experienced higher GRFr magnitudes than IHC subjects at both events; mean GRFr at impact peak $=92 \%$ BW in FFC and $70 \%$ BW in IHC, and at braking peak $=91 \%$ BW in FFC and 69\% BW in IHC (Table 2). Normalized Fy forces (Table 2) were not significantly different between FFC and IHC subjects. At touchdown and impact peak in most cases Fy was in a direction indicating a braking force (i.e., a posteriorly directed GRFr). However, in some steps Fy indicated a propulsive value in early stance (i.e., an anteriorly directed GRFr). This suggested that the foot was under the body during that phase (Fy traces are presented in Fig. 2). There were no significant differences in normalized Fy/Fz between FFC and IHC (p > 0.05) at touchdown, impact peak, or braking peak (Table 2). Normalized mediolateral force (Fx) was significantly higher in FFC than IHC at touchdown ( $\mathrm{p}<0.01$ ) (Table 2).

Mean GRFr angles at touchdown, impact peak, and braking peak were calculated in two ways to explore direction in detail: 1) only steps in which the GRFr was posteriorly directed 2) all steps (i.e., posteriorly and anteriorly directed GRFr). At touchdown in FFC, the mean GRFr angle was significantly smaller (i.e., the GRFr was directed less posteriorly) when all steps were considered than when only posterior steps were considered $(\mathrm{p}<0.01)$. However, there were no significant differences in GRFr angle between IHC and FFC at impact peak or braking peak ( $p>0.05)$ (Table 2). Even when all steps were considered at touchdown in FFC, the mean GRFr angle was still greater than $90^{\circ}$ (Table 2), thus, the predominant direction of the GRFr at touchdown, impact peak, and braking peak was posterior and the mean GRFr angle was $>90^{\circ}$ in both IHC and FFC (Fig. 3).

\section{Discussion}

This study documents the loading patterns of initial heel contact (IHC) and flat foot contact (FFC) in infant/toddler walking. IHC steppers are older and heavier, with longer legs and more walking experience than FFC steppers. The IHC is characterized by the COP under the heel during significant loading events proportionately more frequently than observed during FFC.

Calcaneal loading is, in part, a function of the position of the foot relative to the body, not position of the foot relative to the leg (i.e., ankle angle). Contrary to predictions, ankle angles did not differ between foot contact types. Yet, as predicted, and as reported by plantar pressure studies $[4,5]$, the COP was consistently under the heel in those children who use an IHC as it is in adults [17-20], whereas in children who land with a FFC, the COP was frequently located anterior to the heel at touchdown. Following initial heel contact in IHC steps, the COP often remained under the heel throughout impact and braking peak. Thus, IHC steps represent a transitional heel loading pattern from flat foot contact to that of adults, who maintain an even longer period with the COP under the heel, resulting in even longer periods of heel loading [17-21].

Lower vertical forces at impact in IHC walkers compared to FFC walkers likely reflect the transition to a heel-to-toe rocker pattern or the use of knee yield to absorb peak forces. In adults, slight knee yield 
follows heel strike, and plays an important role in absorbing energy and offsetting impact loads [28]. FFC steppers experienced significantly higher vertical forces at impact peak and at braking peak than IHC, reflecting the rapid downward trajectory of the foot and leg in FFC. In the youngest children, co-activation of antagonist leg muscles prior to two years of age stiffens the legs and may limit lower limb joint movements [29]. Thus, children who used FFC and experienced high impact transients may have used a less yielding gait. Hallemans and colleagues [30] reported a less yielding gait in children with between 2 weeks and 5 months walking experience compared to adults, suggesting that the knee did not yield or absorb energy in children [30].

Compared to IHC children, young FFC children with a relatively wider heel experienced significantly higher, more variable mediolateral forces at touchdown, suggesting a form-function relationship among mediolateral force and heel width. Yet, overall, mediolateral GRFs were low compared to vertical and braking GRFs. Although FFC subjects stepped down with a fully plantigrade foot and experienced higher vertical forces, they applied similar braking forces as a percentage of vertical force as IHC subjects. As a result, while GRFr angles were often posteriorly oriented in both FFC and IHC steps, FFC walkers experienced significantly higher GRFr magnitude at impact peak and braking peak. Increased impact transients and GRFr magnitudes, coupled with a relatively stiff limb and the presence of the COP under the calcaneus in some FFC steps ( $8 \%$ of steps at impact peak), may be a contributor to calcaneal injuries and overuse damage in some very early walkers [10-12].

From the earliest steps, the heel of young walkers experiences high, cyclical impact forces. Impact transients appear as early as 11.5 months of age with the first FFC steps. At this young age, the COP is not always under the heel and high magnitude vertical forces are likely distributed across the flat foot, with at least some force applied to the calcaneus. The wide heel (heel pad and additional soft tissue) of FFC subjects may help mitigate this loading in early walkers with incomplete formation of the calcaneus. In contrast, a relatively narrow heel pad in children who use IHC reduces the surface area over which GRFs can be distributed and, combined with more direct and longer duration calcaneal loading, may require a wider bony base of the calcaneal tuber to absorb GRFs at initial heel contact. But this prediction is contradicted by the data presented here showing that the average position of the COP under the heel does not correspond to the bony medial or lateral plantar processes of the calcaneal tuber. Thus, rather than the lateral plantar process, the more anterior aspects of the calcaneus and heel pad may experience (and possibly mediate) high ground reaction forces during early stance in young children. This observation suggests a possible explanation for injury patterns in young children and provides a foundation for interpreting form-function relationships in future studies of calcaneal and heel pad development in children.

\section{Conflict of interest statement}

There are no conflicts of interest associated with this research.

\section{Acknowledgements}

We thank Chris Kirk, John Kappelman, Denné Reed, and Brian Richmond for their comments on an earlier version of this manuscript. We are also grateful to three anonymous reviewers for their constructive comments. Thanks also to Amber N. Heard-Booth, Kelly Manrriquez, Graham Noblit, Perla Santillan, and Renate Van ZandwijkRowley for assistance. This research was supported by the Leakey Foundation (A.Z.) and an NSF Doctoral Dissertation Improvement Grant (BCS-1028958 to A.Z.).

\section{References}

[1] B. Latimer, C.O. Lovejoy, The calcaneus of Australopithecus afarensis and its implications for the evolution of bipedality, Am. J. Phys. Anthropol. 78 (1989) 369-386.

[2] C. Bertsch, H. Unger, W. Winkelmann, D. Rosenbaum, Evaluation of early walking patterns from plantar pressure distribution measurements: first year results of 42 children, Gait Posture 19 (2004) 235-242.

[3] H. Forssberg, Ontogeny of human locomotor control I. Infant stepping, supported locomotion and transition to independent locomotion, Exp. Brain Res. 57 (1985) 480-493.

[4] A. Hallemans, K. D’Aout, D. De Clercq, P. Aerts, Pressure distribution patterns under the feet of new walkers: the first two months of independent walking, Foot Ankle Int. 24 (2003) 444-453.

[5] A. Hallemans, D. De Clercq, S.V. Dongen, P. Aerts, Changes in foot function parameters during the first 5 months after the onset of independent walking: a longitudinal follow-up study, Gait Posture 23 (2006) 142-148.

[6] B.M. Myklebust, A review of myotatic reflexes and the development of motor control and gait in infants and children: a special communication, Phys. Ther. 70 (1990) 188-203.

[7] C.N. Burnett, E.W. Johnson, Development of gait in childhood: part II, Dev. Med. Child Neurol. 13 (1971) 207-215.

[8] S. Preis, A. Klemms, K. Muller, Gait analysis by measuring ground reaction forces in children: changes to an adaptive pattern between the ages of one and five years, Dev. Med. Child Neurol. 39 (1997) 228-233.

[9] E. Thelen, B. Bril, Y. Breniere, The emergence of heel strike in newly walking infants: a dynamic interpretation, in: F. Horak (Ed.), In Posture and Gait: Control Mechanisms, University of Oregon Books, Eugene, 1992, pp. 334-337.

[10] R.J. Starshak, G.W. Simons, J.R. Sty, Occult fracture of the calcaneus- another toddler's fracture, Pediatr. Radiol. 14 (1984) 37-40.

[11] J.A. Ogden, T.M. Ganey, J.D. Hill, J.I. Jaakkola, Sever's injury: a stress fracture of the immature calcaneal metaphysis, J. Pediatr. Orthop. 24 (2004) 488-492.

[12] C.L. Hendrix, Calcaneal apophysitis (Sever disease), Clin. Podiatr. Med. Surg. 22 (1) (2005) 55-62.

[13] K.-J. Chi, D. Schmitt, Mechanical energy and effective foot mass during impact loading of walking and running, J. Biomech. 38 (2005) 1387-1395.

[14] K. Gruber, H. Ruder, J. Denoth, K. Schneider, A comparative study of impact dynamics: wobbling mass model versus rigid body models, J. Biomech. 31 (1998) 439-444.

[15] D.E. Lieberman, M. Venkadesan, W.A. Werbel, A.I. Daoud, S. D'Andrea, I.S. Davis, R.O. Mang'Eni, Y. Pitsiladis, Foot strike patterns and collision forces in habitually barefoot versus shod runners, Nature 463 (2010) 531-535.

[16] M.W. Wittle, Generation and attenuation of transient impulsive forces beneath the foot: a review, Gait Posture 10 (1999) 264-275.

[17] J.M. Czerniecki, Foot and ankle biomechanics in walking and running: a review, Am. J. Phys. Med. Rehabil. 67 (6) (1988) 246-252.

[18] H. Elftman, A cinematic study of the distribution of pressure in the human, Anat. Rec. 59 (1934) 481-491.

[19] H. Elftman, J. Manter, Chimpanzee and human feet in bipedal walking, Am. J. Phys. Anthropol. 20 (1935) 69-79.

[20] M. Grundy, P.A. Tosh, R.D. McLeish, L. Smidt, An investigation of the centres of pressure under the foot while walking, J. Bone Joint Surg. 57 (1975) 98-103.

[21] A. Hallemans, D. De Clercq, P. Aerts, Changes in 3D joint dynamics during the first 5 months after the onset of independent walking: a longitudinal follow-up study, Gait Posture 24 (2006) 270-279.

[22] K.E. Adolf, S.E. Berger, Motor Development. In Damon and Lerner (Series Editors), in: Kuhn, Seigler (Eds.), Handbook of Child Psychology Volume 2 Cognition, Perception, and Language, Wiley, New York, 2017, pp. 161-213.

[23] L.W. Cowgill, A. Warrener, H. Pontzer, C. Ocobock, Waddling and toddling: the biomechanical effects of an immature gait, Am. J. Phys. Anthropol. 143 (2010) $52-61$.

[24] J. Stebbins, M. Harrington, N. Thompson, A. Zavatsky, T. Theologis, Repeatability of a model for measuring multi-segment foot kinematics in children, Gait Posture 23 (2006) 401-410.

[25] D. Winter, Biomechanics and Motor Control of Human Movement, Hoboken, John Wiley and Sons, Inc, 2005.

[26] J.L. Jensen, B.D. Ulrich, E. Thelen, K. Schneider, R.F. Zernicke, Adaptive dynamics in the leg movement patterns of human infants: i: The effects of posture on spontaneous kicking, J. Motor Behav. 26 (1994) 303-312.

[27] B.D. Ulrich, J.L. Jensen, E. Thelen, K. Schneider, R.F. Zernicke, Adaptive dynamics of the leg movement patterns of human infants: II: treadmill stepping in infants and adults, J. Motor Behav. 26 (1994) 313-324.

[28] J.E. Clark, S.J. Phillips, The step cycle organization of infant walkers, J Motor Behav. 19 (1987) 421-433.

[29] P.S.C. Chagas, M.C. Mancini, S.T. Fonseca, T.B.C. Soares, V.P.D. Gomes, R.F. Sampaio, Neuromuscular mechanics and anthropometric modifications in the initial stages of independent walking, Gait Posture 24 (2006) 375-381.

[30] A. Hallemans, D. De Clercq, B. Otten, P. Aerts, 3D joint dynamics of waking in toddlers: a cross-sectional study spanning the first rapid development phase of walking, Gait Posture 22 (2005) 107-118. 